\title{
Borboletas (Lepidoptera: Hesperioidea e Papilionoidea) do campus da Universidade Federal de Santa Maria, Santa Maria, Rio Grande do Sul
}

\author{
Gabriel Dorneles Sackis ${ }^{1,2}$; Ana Beatriz Barros de Morais ${ }^{1}$ \\ ${ }^{1}$ Laboratório de Interações Inseto-Planta, Departamento de Biologia, \\ Centro de Ciências Naturais e Exatas, Universidade Federal de Santa Maria - UFSM, \\ Faixa de Camobi, Km 09, CEP 97105-900, Santa Maria, RS, Brasil \\ ${ }^{2}$ Autor para correspondência: Gabriel Dorneles Sackis, e-mail: gsackis@yahoo.com.br
}

Sackis, G. D.; Morais, A. B. B. Butterflies (Lepidoptera: Hesperioidea and Papilionoidea) from Universidade Federal de Santa Maria campus, Santa Maria, Rio Grande do Sul. Biota Neotrop., vol. 8, no. 1, Jan./Mar. 2008. Available from: <http://www.biotaneotropica.org.br/v8n1/en/abstract?inventory+bn01908012008>.

\begin{abstract}
This work aimed to investigate the composition, richness and diversity of butterflies from Universidade Federal de Santa Maria - UFSM, campus area of Camobi, Santa Maria, Rio Grande do Sul. The field work was performed bimonthly from September 2005 to September 2006, with standardized effort of seven hours/entomological net/occasion. 872 individuals from 89 species were registred on 113 hours: 575 (65.9\%) from Nymphalidae, 174 (19.9\%) from Hesperiidae, 88 (10.1\%) Pieridae, 21 (2.4\%) Lycaenidae, 10 (1.2\%) Papilionidae and four $(0.5 \%)$ Riodinidae. 40 (44.9\%) species were from Nymphalidae, $25(28.1 \%)$ Hesperiidae, 11 (12.4\%) Pieridae, 8 (8.9\%) Lycaenidae, three (3.4\%) Papilionidae and two (2.3\%) Riodinidae. The five most abundant species from Camobi campus were Pyrgus orcus (Stoll, 1780) (N=78) (Hesperiidae) and Nymphalidae Hermeuptychia hermes (Fabricius, 1775) $(\mathrm{N}=70)$, Anartia amathea roeselia (Eschscholtz, 1821) $(\mathrm{N}=55)$, Vanessa braziliensis (Moore, 1883) $(\mathrm{N}=51)$ and Heliconius erato phyllis (Fabricius, 1775) $(\mathrm{N}=51)$. Only H. hermes and $V$. braziliensis, typical from grasslands and open habitats, are not characteristic of disturbed environments. Previous studies registered 12 more butterfly species, raising local species richness to 101. Although strongly human impacted, the Camobi campus is still able to sustain an expressive butterfly fauna.

Keywords: butterfly, conservation, diversity, Pampa biome, species richness.
\end{abstract}

Sackis, G. D.; Morais, A. B. B. Borboletas (Lepidoptera: Hesperioidea e Papilionoidea) do campus da Universidade Federal de Santa Maria, Santa Maria, Rio Grande do Sul. Biota Neotrop., vol. 8, no. 1, jan./mar. 2008. Disponível em: <http://www.biotaneotropica.org.br/v8n1/pt/abstract?inventory+bn01908012008>.

Resumo: Este trabalho teve por objetivo investigar a composição, riqueza e diversidade das borboletas encontradas na área do campus Camobi da Universidade Federal de Santa Maria - UFSM, Santa Maria, Rio Grande do Sul. As amostragens foram realizadas bimestralmente de setembro de 2005 a setembro de 2006, com esforço amostral padronizado em cerca de sete horas/rede entomológica/ocasião. No total de 113 horas de amostragem, foram registrados 872 indivíduos, distribuídos em 89 espécies. Do total de indivíduos, $575(65,9 \%)$ pertencem à família Nymphalidae, 174 (19,9\%) a Hesperiidae, 88 (10,1\%) Pieridae, 21 (2,4\%) Lycaenidae, 10 (1,2\%) Papilionidae e quatro $(0,5 \%)$ Riodinidae. Do total de espécies, $40(44,9 \%)$ pertencem à família Nymphalidae, $25(28,1 \%)$ à Hesperiidae, 11 (12,4\%) Pieridae, oito (8,9\%) Lycaenidae, três (3,4\%) Papilionidae e duas (2,3\%) Riodinidae. As cinco espécies mais abundantes do campus Camobi foram Pyrgus orcus (Stoll, 1780) ( $\mathrm{N}=78$ ) (Hesperiidae) e os Nymphalidae Hermeuptychia hermes (Fabricius, 1775) ( $\mathrm{N}=70)$, Anartia amathea roeselia (Eschscholtz, 1821) ( $\mathrm{N}=55)$, Vanessa braziliensis (Moore, 1883) $(\mathrm{N}=51)$ e Heliconius erato phyllis (Fabricius, 1775) $(\mathrm{N}=51)$. Destas, apenas $H$. hermes e $V$. braziliensis, consideradas comuns em campos ou ambientes abertos, não são características de ambientes perturbados. Estudos anteriores registraram mais 12 espécies de borboletas, elevando a riqueza local de espécies para 101. Apesar de estar sujeito a forte ação antrópica, o campus Camobi ainda é capaz de abrigar uma fauna expressiva de borboletas.

Palavras-chave: bioma Pampa, borboleta, conservação, diversidade, riqueza de espécies. 


\section{Introdução}

A ordem Lepidoptera constitui um grupo de insetos muito diversificado e bem sucedido ecologicamente. As mariposas representam a grande maioria dos insetos desta ordem, com cerca de 127.000 (87\%) espécies descritas, distribuídas em 25 superfamílias. Já as borboletas, objeto de estudo do presente trabalho, apresentam cerca de 19.000 (13\%) espécies descritas, distribuídas em duas superfamílias: Hesperioidea e Papilionoidea (Heppner 1991, Brown Jr. \& Freitas 1999). O termo popular borboleta refere-se àqueles lepidópteros de hábitos diurnos e crepusculares, com representantes distribuídos em seis famílias: Hesperiidae, Papilionidae, Pieridae, Lycaenidae, Riodinidae e Nymphalidae (Wahlberg et al. 2005). No Brasil, existem aproximadamente entre 3.130 e 3.300 espécies descritas de borboletas (Beccaloni \& Gaston 1995, Brown Jr. \& Freitas 1999). Por ter sua sistemática relativamente bem conhecida, as borboletas são objetos de investigação em diversos estudos biológicos, dentre eles: interações inseto-planta, conservação de habitats naturais, variabilidade genética em populações, biogeografia e uso como bioindicadores, entre outros (Brown Jr. \& Freitas 1999, Freitas et al. 2006).

A maior parte dos estudos de inventariamento de borboletas no Brasil foi feita em latitude tropical (Brown Jr. \& Freitas 1999), principalmente nas regiões Norte e Sudeste. No Rio Grande do Sul, as primeiras informações sobre a fauna de borboletas são, muitas vezes, incompletas em relação aos locais estudados, períodos de coleta e esforço amostral empregado. Atualmente, devido ao crescente número de trabalhos desenvolvidos e ao maior conhecimento da biologia desses insetos, as publicações mostram-se bastante detalhadas. Dentre as mais recentes, destacam-se Iserhard \& Romanowski (2004) em floresta de mata atlântica, na região nordeste do estado e Paz (2005) na Serra do Sudeste, em vegetação do tipo campos sulinos. Ainda, Marchiori \& Romanowski (2006a) avaliaram a composição e deslocamentos diários de borboletas, em vegetação do tipo mata de restinga no Parque Estadual de Itapuã, localizado próximo ao município de Porto Alegre; e no Parque Estadual do Espinilho e entorno, região centro-oeste, onde Marchiori \& Romanowski (2006b) trabalharam em vegetação do tipo savana estépica parque e mata ciliar. Na região central do estado, Dessuy \& Morais (2007) estudaram a fauna de borboletas em três áreas distintas de Santa Maria cobertas por matas naturais do tipo Floresta Estacional Decidual.

Na região de Santa Maria, os primeiros inventários foram feitos por Link et al. (1977, 1980), com registro apenas de espécies pertencentes às famílias Papilionidae, Pieridae e subfamílias Morphinae e Brassolinae (Nymphalidae). Kaminski (1992) registrou e avaliou coleções de insetos de Santa Maria e seus arredores, incluindo espécies de borboletas de todas as famílias, exceto Lycaenidae. Schwartz \& Di Mare (2001) e Paim \& Di Mare (2002) realizaram estudos sobre diversidade e ecologia de papilionídeos, os primeiros analisando a diversidade de 15 espécies em sete áreas distintas de Santa Maria, incluindo ambientes urbanos, e os últimos relatando parâmetros biológicos e demográficos de uma única espécie, Parides agavus (Drury, 1782). O primeiro trabalho completo de inventariamento de borboletas nessa região foi realizado por Dessuy \& Morais (2007), conforme anteriormente mencionado.

As modificações humanas na paisagem e o processo de urbanização têm levado à destruição, fragmentação e ao isolamento de habitats naturais, com conseqüente prejuízo para a biodiversidade (Fahrig 2003) e aumentando a importância das áreas remanescentes como refúgio para a fauna e flora. Em relação às comunidades de borboletas, já existem trabalhos de inventariamento indicando a importância e efetividade desses habitats para manutenção da fauna regional (Brown Jr. \& Freitas 2002, Collier et al. 2006). Reservas, parques, jardins e/ou outros tipos de áreas verdes, localizados dentro das cidades ou em seus arredores, podem oferecer um mosaico de recursos de alimentação, oviposição, abrigo e permanência para muitas espécies de borboletas (Brown Jr. \& Freitas 2002). Tem sido proposto a re-vegetação dos parques urbanos com espécies de plantas hospedeiras potenciais e outros tipos de recursos de modo a incrementar o valor dessas áreas para conservação de borboletas e outros grupos faunísticos (Brown Jr. \& Freitas 2002, Koh \& Sodhi 2004). O presente trabalho tem por objetivo investigar a composição, riqueza e diversidade das borboletas encontradas na área do campus Camobi da Universidade Federal de Santa Maria (UFSM), município de Santa Maria, Rio Grande do Sul (RS), contribuindo para o conhecimento da fauna de lepidópteros diurnos da região e do estado.

\section{Material e Métodos}

\section{1. Área de estudo}

A cidade de Santa Maria localiza-se na região central do estado do Rio Grande do Sul. A vegetação faz parte da Floresta Estacional Decidual da Fralda da Serra Geral (Pereira et al. 1989), ocupando uma zona de transição entre a Depressão Central do estado e a escarpa basáltica do Planalto Meridional Brasileiro, no domínio do bioma Pampa. O clima é temperado chuvoso e quente, do tipo Cfa com nevoeiros. Os índices pluviométricos anuais variam entre $1.500 \mathrm{e}$ $1.700 \mathrm{~mm}$, sendo maio e junho os meses de maior precipitação. As temperaturas médias mínimas ficam entre $8^{\circ}$ e $10^{\circ} \mathrm{C}$ e as máximas entre $32^{\circ} \mathrm{e} 40^{\circ} \mathrm{C}$ (Pereira et al. 1989). O campus Camobi da UFSM, cuja altitude é de cerca de $95 \mathrm{~m}$, localiza-se no bairro Camobi, na região leste da cidade $\left(29^{\circ} 42^{\prime} \mathrm{S}\right.$ e $\left.53^{\circ} 42^{\prime} \mathrm{W}\right)$ e fica distante cerca de 12 km do centro, próximo à rodovia RS 509, saída para a capital Porto Alegre. Possui uma área de aproximadamente 1907 ha e foi criado em 1960 numa região que abrangia principalmente área de campo e mata ciliar (Dambros et al. 2004). A vegetação predominante é campo, com algumas áreas de Pinus e Eucalyptus, além de um arroio e açudes (Ethur et al. 1995), e a presença de um Jardim Botânico, com área de 13 ha e aproximadamente 800 árvores, além de ervas, num total de 515 espécies, pertencentes a 96 famílias. Atualmente, as diferentes fisionomias do campus apresentam alterações decorrentes da forte ação antrópica (Dambros et al. 2004, Santos et al. 2005).

\subsection{Amostragem}

As borboletas foram amostradas bimestralmente, com esforço de aproximadamente sete horas/rede entomológica/ocasião, no período de setembro de 2005 a setembro de 2006 . Foram percorridas trilhas e/ou estradas de terra por diversos ambientes da área do campus, incluindo o interior do Jardim Botânico, no horário compreendido entre 8:30 e 15:30 horas, período de maior atividade das borboletas. Essas trilhas foram consideradas transectos e percorridas uma única vez, em cada ocasião amostral, por dois a três observadores experientes. Todas as borboletas visualizadas e identificadas foram registradas e algumas, cuja espécie não foi possível determinar no campo, foram coletadas para análise posterior em laboratório. A identificação dos espécimes coletados foi realizada com auxílio de bibliografia especializada (Brown Jr. 1992, Canals 2000, 2003 - fauna da Argentina, províncias de Buenos Aires e Misiones, D’Abrera 1988, 1994, 1995, DeVries 1987, 1997 - fauna da Costa Rica, Tyler et al. 1994) e consulta a especialistas e à coleção entomológica do Departamento de Zoologia, Instituto de Biociências, da Universidade Federal do Rio Grande do Sul. Os indivíduos que não puderam ser identificados em campo ou em laboratório foram desconsiderados. A nomenclatura taxonômica foi atualizada de acordo com Lamas (2004) e Mielke (2005). Alguns exemplares foram depositados como referência na coleção de insetos do Laboratório de Interações Inseto- 
Planta do Departamento de Biologia, Centro de Ciências Naturais e Exatas da Universidade Federal de Santa Maria, Santa Maria, RS. A amostragem utilizada no presente trabalho está de acordo com a metodologia proposta no projeto "As Borboletas do Rio Grande do Sul” (Iserhard \& Romanowski 2004).

\subsection{Análise dos dados}

Foram analisados os seguintes itens: número de indivíduos registrados $(\mathrm{N})$, total e por ocasião amostral; riqueza $(\mathrm{S})$ total e por ocasião amostral; e curva cumulativa de espécies, visando obter o número cumulativo de espécies novas durante as amostragens. Foi calculado o índice de constância das espécies amostradas (Bodenheimer 1955, apud Schwartz \& Di Mare 2001), o qual expressa a percentagem de espécies presentes nas ocasiões amostrais, através da fórmula $\mathrm{C}=\mathrm{p} \times 100 / \mathrm{N}(\mathrm{p}=$ ocasiões amostrais contendo a espécie estudada e $\mathrm{N}=$ total de ocasiões amostrais), resultando as seguintes categorias: a) Constantes (C), presentes em mais de 50\% das ocasiões amostrais, b) Acessórias (A), presentes em 25\% a 50\% das ocasiões amostrais e c) Acidentais (Ac), presentes em menos de $25 \%$ das ocasiões amostrais. A frequiência relativa (fr) foi obtida pela razão entre o número de indivíduos de uma espécie e o número total de indivíduos de todas as espécies. Foram consideradas "dominantes" as espécies com frequiência relativa maior que $10 \%$ ( $\mathrm{fr}>0,1$ ) (Dessuy \& Morais 2007). Espécies registradas por um único indivíduo foram denominadas "singletons" (Novotný \& Basset 2000). Também foram calculados os Índices de Diversidade de Shannon-Wiener (H') e de Margalef (Dmg), e Índices de Dominância de Simpson (D) e de Berger-Parker (d), utilizando o programa estatístico Past, versão 1.35b.

\section{Resultados}

Em 113 horas/rede de amostragem, foi registrado um total de 872 indivíduos, distribuídos em 89 espécies de borboletas. Do total de indivíduos, $575(65,9 \%)$ pertencem à família Nymphalidae, $174(19,9 \%)$ Hesperiidae, 88 (10,1\%) Pieridae, 21 (2,4\%) Lycaenidae, $10(1,2 \%)$ Papilionidae e quatro $(0,5 \%)$ Riodinidae (Tabela 1$)$. Do total de espécies, $40(44,9 \%)$ pertencem à família Nymphalidae, $25(28,1 \%)$ à família Hesperiidae, $11(12,4 \%)$ a Pieridae, $8(8,9 \%)$ a Lycaenidae, três $(3,4 \%)$ a Papilionidae e duas $(2,3 \%)$ a Riodinidae (Tabela 1). Das espécies coletadas, duas foram registradas pela primeira vez no estado: o Lycaenidae Laothus phydela (Hewitson, 1867) (campus UFSM, 13.VII.2006, Robbins det.; Sackis leg.) e o Riodinidae Euselasia eucerus (Hewitson, 1872) (campus UFSM, 10.V.2006, Mielke det.; Lemes leg.) (Biezanko 1949, 1960a, b, c, d, Biezanko 1963, Biezanko \& Mielke 1973, Biezanko et al. 1978, Mielke 1979, Teston \& Corseuil 2001, 2002a, b, Kruger \& Silva 2003, Iserhard \& Romanowski 2004, Quadros et al. 2004, Mielke 2005, Marchiori \& Romanowski 2006a, b, Dessuy \& Morais 2007).

As cinco espécies mais abundantes do campus Camobi foram Pyrgus orcus (Stoll, 1780) $(\mathrm{N}=78)$ (Hesperiidae) e os Nymphalidae Hermeuptychia hermes (Fabricius, 1775) $(\mathrm{N}=70)$, Anartia amathea roeselia (Eschscholtz, 1821) $(\mathrm{N}=55)$, Vanessa braziliensis (Moore, 1883) $(\mathrm{N}=51)$ e Heliconius erato phyllis (Fabricius, 1775) $(\mathrm{N}=51)$ (Tabela 1). Trinta e duas espécies foram representadas por um único indivíduo e consideradas "singletons" (Tabela 1).

O número cumulativo de espécies por ocasião de amostragem (Figura 1) manteve-se bastante crescente até a quarta ocasião amostral (março de 2006), porém, a partir daí, a inclinação da curva apresenta-se de forma bastante suave. Isso parece indicar que o esforço amostral empregado na área do campus Camobi atingiu um nível satisfatório.

De acordo com o Índice de Constância, 37 espécies (41\%) são acidentais, 30 (34\%) são acessórias e 22 (25\%) são constantes na área do campus Camobi da UFSM (Tabela 1). No entanto, nenhuma das espécies consideradas constantes se mostrou dominante ( $\mathrm{fr}>0,1$ ) no presente trabalho, nem mesmo as cinco espécies mais abundantes (Figura 2). Porém, P. orcus ( $\mathrm{fr}=0,089)$ e H. hermes $(\mathrm{fr}=0,08)$ apresentaram os valores mais próximos ao limiar de 0,1 . Com relação aos Índices de Diversidade de Shannon-Wiener (H') e de Margalef (Dmg) os valores obtidos foram: H' = 3,6 e Dmg = 13. Enquanto os Índices de Dominância de Simpson (D) e de Berger-Parker (d) apresentaram os valores de $\mathrm{D}=0,04183$ e $\mathrm{d}=0,08945$.

A riqueza de espécies de borboletas encontradas no campus variou entre as ocasiões amostrais e o mês de março apresentou o maior valor $(S=45)$, seguido de janeiro e maio $(S=37)$, devido principalmente às maiores riquezas das famílias Nymphalidae e Hesperiidae (Tabela 2). Já o mês de setembro de 2005 apresentou a menor riqueza $(S=14)$, quando não foram registradas espécies de Papilionidae e Pieridae. De modo geral, os valores de abundância total de indivíduos também acompanharam o padrão das riquezas, com exceção dos meses de maio e janeiro que apresentarem um grande aumento do número de indivíduos, principalmente de Nymphalidae e Hesperiidae (Tabela 3).

\section{Discussão}

Outros estudos realizados no Rio Grande do Sul, em diferentes formações vegetais, utilizando metodologia semelhante à do presente trabalho (Iserhard \& Romanowski 2004, Paz 2005, Marchiori

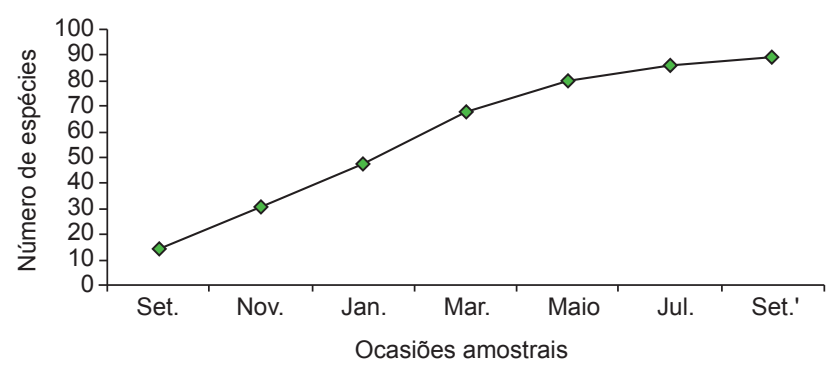

Figura 1. Número cumulativo de espécies de borboletas obtido em sete ocasiões amostrais, na área do campus Camobi da Universidade Federal de Santa Maria, Santa Maria, Rio Grande do Sul, Brasil, no período de setembro de 2005 (set) a setembro de 2006 (set').

Figure 1. Cumulative number of butterfly species registered on seven sampling ocasions, in Universidade Federal de Santa Maria campus area of Camobi, Santa Maria, Rio Grande do Sul, Brazil, from September 2005 (set) to September 2006 (set').

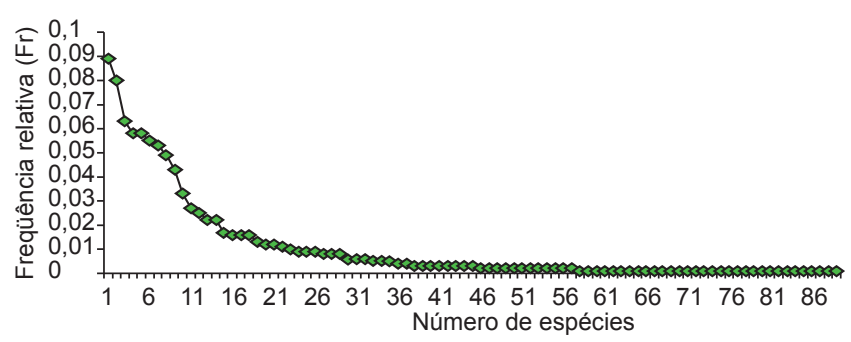

Figura 2. Distribuição das frequências relativas das espécies de borboletas amostradas na área do campus Camobi da Universidade Federal de Santa Maria, Santa Maria, Rio Grande do Sul, Brasil, no período de setembro de 2005 a setembro de 2006 .

Figure 2. Relative frequency distribution of butterfly species sampled in Universidade Federal de Santa Maria campus area of Camobi, Santa Maria, Rio Grande do Sul, Brazil, from September 2005 to September 2006. 
Tabela 1. Espécies de borboletas registradas na área do campus da Universidade Federal de Santa Maria - UFSM, Santa Maria, Rio Grande do Sul, Brasil, de setembro de 2005 a setembro de 2006. N: total de indivíduos, S: riqueza de espécies, * : espécies representadas por um único indivíduo ("singletons"), Ac: espécies acidentais, A: espécies acessórias, C: espécies constantes $\mathrm{e}^{+}$: espécies registradas em estudos prévios (Lemes et al., dados não publicados) na mesma área.

Table 1. Butterfly species registered in Universidade Federal de Santa Maria campus area of Camobi - UFSM, Santa Maria, Rio Grande do Sul, Brazil, from September 2005 to September 2006. N: total number of individuals, S: species richness, * : species represented as single individuals ("singletons"), Ac: accidental species, A: accessory species, C: constant species and ${ }^{+}$: species registered on previous studies (Lemes et al., data not published) at the same area.

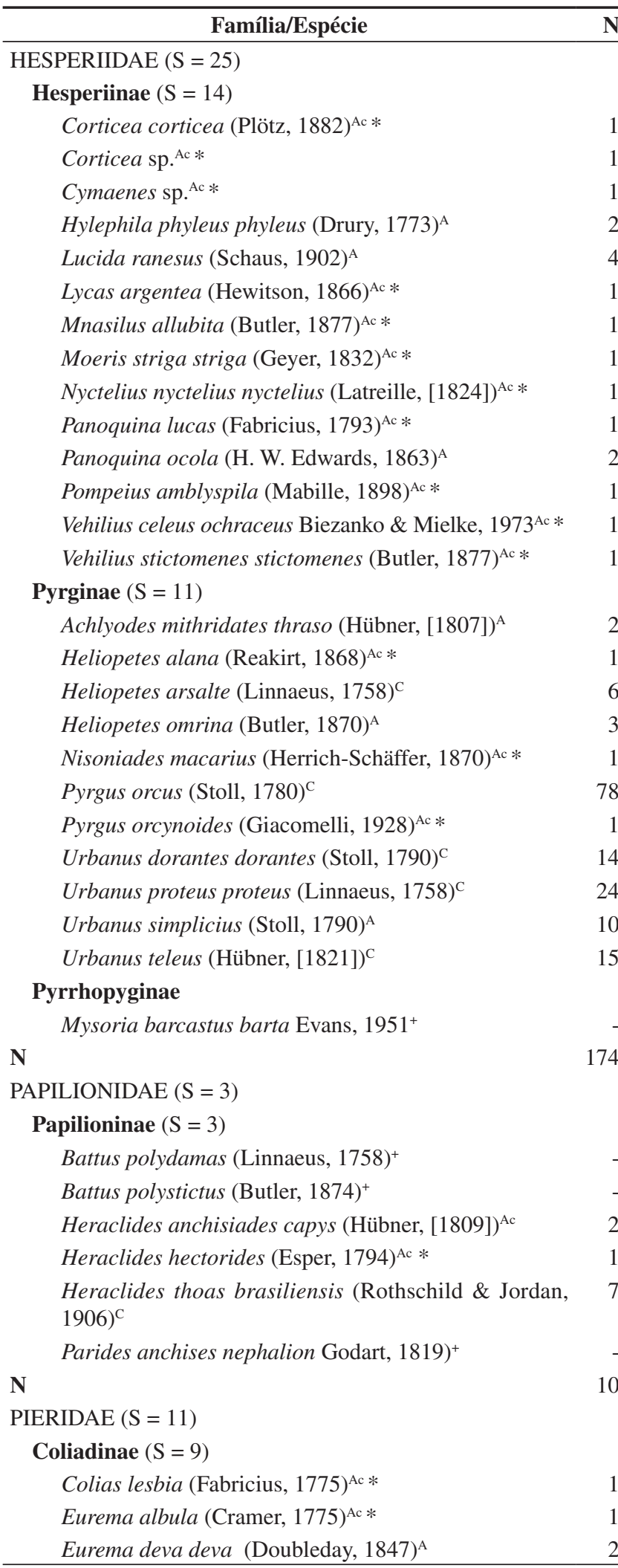

$\mathbf{N}$

1

1

1

2

\begin{tabular}{|c|c|}
\hline Família/Espécie & $\mathbf{N}$ \\
\hline Eurema elathea elathea $\left(\right.$ Cramer, 1777) ${ }^{\mathrm{C}}$ & 11 \\
\hline Eurema phiale paula (Röber, 1909) ${ }^{\mathrm{Ac} *}$ & 1 \\
\hline Phoebis neocypris neocypris (Hübner, [1823]) ${ }^{\mathrm{C}}$ & 20 \\
\hline Phoebis philea philea (Linnaeus, 1763) ${ }^{\mathrm{C}}$ & 22 \\
\hline Phoebis sennae marcellina $\left(\right.$ Cramer, 1777) ${ }^{\mathrm{C}}$ & 8 \\
\hline Pyrisitia nise tenella (Boisduval, 1836) ${ }^{\mathrm{A}}$ & 7 \\
\hline \multicolumn{2}{|l|}{ Pierinae $(S=1)$} \\
\hline Ascia monuste orseis (Godart, 1819) ${ }^{\mathrm{A}}$ & 14 \\
\hline \multicolumn{2}{|l|}{ Dismorphiinae $(S=1)$} \\
\hline Enantia sp. $^{\text {Ac. } *}$ & 1 \\
\hline Enantia lina (Herbst, 1792)+ & - \\
\hline $\mathbf{N}$ & 88 \\
\hline
\end{tabular}

LYCAENIDAE $(\mathrm{S}=8)$

Theclinae $(\mathrm{S}=7)$

Arawacus separata Lathy, 1926 ${ }^{\mathrm{A}} 5$

Calycopis caulonia (Hewitson, 1877) ${ }^{\mathrm{A}} \quad 3$

Laothus phydela (Hewitson, 1867) ${ }^{\mathrm{Ac}}$

Panthiades hebraeus (Hewitson, 1867) ${ }^{\mathrm{Ac} *} \quad 1$

Parrhasius orgia (Hewitson, 1867) ${ }^{\mathrm{A}} \quad 2$

Rekoa palegon Cramer, 1780

Strymon eurytulus (Hübner, [1819]) ${ }^{\mathrm{A}}$

Polyommatinae $(\mathrm{S}=1)$

Leptotes cassius (Cramer, 1775) ${ }^{\mathrm{A}} \quad 2$

$\mathbf{N}$

RIODINIDAE $(\mathrm{S}=2)$

Euselasiinae (1)

Euselasia eucerus (Hewitson, 1872) Ac * 1

Riodininae (1)

Riodina lycisca (Hewitson, [1853]) ${ }^{\mathrm{A}} 3$

$\mathbf{N}$

NYMPHALIDAE $(\mathrm{S}=40)$

Heliconiinae $(\mathrm{S}=11)$

Actinote carycina Jordan, 1913 ${ }^{\mathrm{A}} 5$

Actinote mamita mamita (Burmeister, 1861) ${ }^{\mathrm{Ac} *} \quad 1$

Actinote melanisans Oberthür, 1917 ${ }^{\mathrm{A}} 5$

Actinote pellenea Hübner, [1821] ${ }^{\mathrm{Ac} *} \quad 1$

Agraulis vanillae maculosa (Stichel, [1908]) ${ }^{\mathrm{C}} 38$

Dione juno juno (Cramer, 1779) ${ }^{\mathrm{A}}$

Dione moneta Hübner, [1825]+

Dryadula phaetusa (Linnaeus, 1758) ${ }^{\mathrm{C}} \quad 29$

Dryas iulia alcionea $\left(\right.$ Cramer, 1779) ${ }^{\mathrm{C}} \quad 48$

Euptoieta claudia claudia (Cramer, 1775) ${ }^{\mathrm{A}} 6$

Heliconius erato phyllis (Fabricius, 1775) ${ }^{\mathrm{C}}$

Heliconius ethilla narcaea Godart, 1819

Nymphalinae ( $\mathrm{S}=10)$

Anartia amathea roeselia $\left(\right.$ Eschscholtz, 1821) ${ }^{\mathrm{C}}$

\begin{tabular}{l}
5 \\
1 \\
\hline \\
\hline \\
\hline \\
6 \\
\hline \\
\hline
\end{tabular}

N
1
20
8
7
14
1
-
8



(1)

1

2

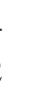

2

(1)

1

3

5


Tabela 1. Continuação..

\begin{tabular}{|c|c|}
\hline Família/Espécie & $\mathbf{N}$ \\
\hline Eresia lansdorfi (Godart, 1819) ${ }^{\mathrm{Ac} *}$ & 1 \\
\hline Hypanartia lethe (Fabricius, 1793) ${ }^{\mathrm{Ac}}$ & 3 \\
\hline Junonia evarete (Cramer, 1779) ${ }^{\mathrm{C}}$ & 47 \\
\hline Ortilia ithra (W. F. Kirby, 1900) ${ }^{\mathrm{A}}$ & 7 \\
\hline Siproeta epaphus trayja Hübner, [1823] ${ }^{\mathrm{A}}$ & 2 \\
\hline Siproeta stelenes meridionalis (Fruhstorfer, 1909)+ & - \\
\hline Tegosa claudina $\left(\right.$ Eschscholtz, 1821) ${ }^{\mathrm{C}}$ & 8 \\
\hline Tegosa orobia (Hewitson, 1864) ${ }^{\mathrm{A}}$ & 3 \\
\hline Vanessa braziliensis (Moore, 1883) ${ }^{\mathrm{C}}$ & 51 \\
\hline Vanessa myrinna (Doubleday, 1849) ${ }^{\mathrm{Ac} *}$ & 1 \\
\hline \multicolumn{2}{|l|}{ Satyrinae $(S=7)$} \\
\hline Capronnieria galesus (Godart, [1824]) ${ }^{\mathrm{Ac} *}$ & 1 \\
\hline Hermeuptychia hermes (Fabricius, 1775) ${ }^{\mathrm{C}}$ & 70 \\
\hline Moneuptychia soter (Butler, 1877) ${ }^{\mathrm{Ac} *}$ & 1 \\
\hline Paryphthimoides poltys (Prittwitz, 1865) ${ }^{\mathrm{A}}$ & 12 \\
\hline Praepedaliodes phanias (Hewitson, 1862)+ & - \\
\hline Taydebis peculiaris (Butler,1874) ${ }^{\mathrm{Ac} *}$ & 1 \\
\hline Yphthimoides celmis $\left(\right.$ Godart, [1824]) ${ }^{\mathrm{C}}$ & 43 \\
\hline Yphthimoides straminea (Butler, 1867) ${ }^{\mathrm{A}}$ & 3 \\
\hline \multicolumn{2}{|l|}{ Biblidinae $(S=5)$} \\
\hline Biblis hyperia nectanabis (Fruhstorfer, 1909) ${ }^{\mathrm{A}}$ & 9 \\
\hline Diaethria candrena candrena (Godart, [1824]) $)^{\mathrm{Ac} *}$ & 1 \\
\hline Eunica eburnea Fruhstorfer, $1907^{\mathrm{A}}$ & 11 \\
\hline Hamadryas epinome (C. Felder \& R. Felder, 1867) ${ }^{\mathrm{Ac}}$ & 3 \\
\hline Hamadryas februa februa (Hübner,[1823]) ${ }^{\mathrm{A}}$ & 8 \\
\hline \multicolumn{2}{|l|}{ Limenitidinae $(S=3)$} \\
\hline Adelpha mincia Hall, 1938 & 2 \\
\hline Adelpha mythra (Godart, [1824]) $)^{\mathrm{Ac} *}$ & 1 \\
\hline Adelpha zea (Hewitson, 1850) (Ac * $^{\text {A }}$ & 1 \\
\hline \multicolumn{2}{|l|}{ Ithomiinae $(\mathrm{S}=2)$} \\
\hline Epityches eupompe (Geyer, 1832) ${ }^{\mathrm{A}}$ & 3 \\
\hline Mechanitis lysimnia lysimnia (Fabricius, 1793) ${ }^{+}$ & - \\
\hline Methona themisto (Hübner, 1818) ${ }^{+}$ & - \\
\hline Pseudoscada erruca (Hewitson, 1855) ${ }^{\mathrm{A}}$ & 2 \\
\hline \multicolumn{2}{|l|}{ Charaxinae $(\mathrm{S}=1)$} \\
\hline Zaretis itys itylus (Westwood, 1850) ${ }^{\mathrm{Ac} *}$ & 1 \\
\hline \multicolumn{2}{|l|}{ Danainae $(S=1)$} \\
\hline Danaus erippus $(\text { Cramer, } 1775)^{\mathrm{C}}$ & 6 \\
\hline \multicolumn{2}{|l|}{ Brassolinae } \\
\hline Blepolenis batea (Hübner, [1821])+ & - \\
\hline \multirow[t]{3}{*}{ Brassolis sophorae (Linnaeus, 1758) ${ }^{+}$} & - \\
\hline & 575 \\
\hline & 872 \\
\hline
\end{tabular}

\& Romanowski 2006a, b), também encontraram Nymphalidae e Hesperiidae como as primeira e segunda famílias mais ricas em espécies, respectivamente. Esses resultados, entretanto, diferem daqueles registrados para outras regiões do Brasil, que indicam Lycaenidae (incluindo Riodinidae) como a família mais representativa (Brown Jr. \& Freitas 1999, Wahlberg et al. 2005). É importante lembrar, porém, a grande diferença entre o esforço amostral empregado no presente trabalho daquele realizado por Brown Jr. \& Freitas (1999), o qual apresenta resultados derivados de vários anos e diferentes métodos de amostragem, realizados principalmente em regiões de latitude mais tropical. No Rio Grande do Sul, parece existir uma tendência a
Tabela 2. Riqueza de espécies registradas por famílias de borboletas por ocasião amostral na área do campus Camobi da Universidade Federal de Santa Maria, Santa Maria, Rio Grande do Sul, Brasil, de setembro de 2005 (Set) a setembro de 2006 (Set').

Table 2. Species richness registered per butterfly family by sample occasion in Universidade Federal de Santa Maria campus area of Camobi, Santa Maria, Rio Grande do Sul, Brazil, from September 2005 (Set) to September 2006 (Set').

\begin{tabular}{lrrrrrrr}
\hline & Set & Nov & Jan & Mar & Mai & Jul & Set' \\
\hline Hesperiidae & 4 & 4 & 7 & 13 & 12 & 4 & 6 \\
Papilionidae & 0 & 1 & 1 & 2 & 1 & 0 & 1 \\
Pieridae & 0 & 3 & 6 & 6 & 5 & 5 & 4 \\
Lycaenidae & 1 & 2 & 3 & 1 & 2 & 2 & 0 \\
Riodinidae & 0 & 1 & 0 & 1 & 2 & 0 & 0 \\
Nymphalidae & 9 & 16 & 20 & 22 & 15 & 15 & 14 \\
$\quad$ Total & 14 & 27 & 37 & 45 & 37 & 26 & 25 \\
\hline
\end{tabular}

Tabela 3. Número de indivíduos registrados por famílias de borboletas por ocasião amostral na área do campus Camobi da Universidade Federal de Santa Maria, Santa Maria, Rio Grande do Sul, de setembro de 2005 (Set) a setembro de 2006 (Set').

Table 3. Number of individuals registered per butterfly family by sample occasion in Universidade Federal de Santa Maria campus area of Camobi, Santa Maria, Rio Grande do Sul, Brazil, from September 2005 (Set) to September 2006 (Set').

\begin{tabular}{lrrrrrrr}
\hline & Set & Nov & Jan & Mar & Mai & Jul & Set' \\
\hline Hesperiidae & 4 & 8 & 28 & 41 & 60 & 18 & 15 \\
Papilionidae & 0 & 2 & 3 & 3 & 1 & 0 & 1 \\
Pieridae & 0 & 9 & 20 & 16 & 14 & 17 & 12 \\
Lycaenidae & 1 & 2 & 6 & 1 & 6 & 5 & 0 \\
Riodinidae & 0 & 1 & 0 & 1 & 2 & 0 & 0 \\
Nymphalidae & 36 & 53 & 125 & 115 & 134 & 50 & 62 \\
$\quad$ Total & 41 & 75 & 182 & 177 & 217 & 90 & 90 \\
\hline
\end{tabular}

um decréscimo de espécies de Lycaenidae em prol de um aumento nas famílias Pieridae, Papilionidae e Nymphalidae, conforme encontrado no presente trabalho e nos outros já mencionados, enquanto Hesperiidae confirmou sua grande representatividade no estado (Morais et al. 2007).

O menor número de espécies de Pieridae encontrado na área do campus Camobi, em relação ao trabalho de Link et al. (1977), o qual registrou 26 espécies, foi provavelmente devido ao uso de diferentes metodologias e maior variação de ambientes amostrados naquele trabalho, incluindo áreas de lavoura. Além disso, esta família pode ter sido subestimada no presente estudo, uma vez que muitos dos indivíduos observados apresentavam um vôo vigoroso, geralmente na altura da copa das árvores, dificultando sua captura e/ou identificação.

As três espécies de Papilionidae, registradas no presente trabalho, pertencem ao gênero Heraclides e duas delas, H. thoas brasiliensis (Rothschild \& Jordan, 1906) e H. anchisiades capys (Hübner, [1809]), ocorrem em lugares perturbados ou em cidades, campos e pomares, respectivamente (Brown Jr.1992, Schwartz \& Di Mare 2001). Em Santa Maria, Schwartz \& Di Mare (2001) e Dessuy \& Morais (2007) listaram, respectivamente, 15 e 12 espécies de papilionídeos em habitats variados, incluindo desde remanescentes de Floresta Decidual da Serra Geral, até jardins eminentemente urbanos.

A composição das espécies mais abundantes de borboletas no campus Camobi foi semelhante à encontrada por Dessuy \& Morais (2007) em fragmentos de Floresta Estacional Decidual de Santa Maria. A maioria é relativamente comum no Rio Grande do Sul 
(Morais et al. 2007), estando associada a campos e florestas perturbadas (Brown Jr. 1992). As exceções foram H. hermes e V. braziliensis características de campos ou ambientes abertos (Brown Jr. 1992) que foram mais abundantes apenas na área do campus, provavelmente porque suas fêmeas ovipositam em gramíneas e compostas herbáceas, respectivamente. Ainda, $V$. braziliensis é a única espécie dentre as mais abundantes do campus que não se encontra relacionada entre as dez mais abundantes do estado do Rio Grande do Sul (Morais et al. 2007). Heliconiinae e Nymphalinae (Nymphalidae) foram as duas subfamílias com maior riqueza e abundâncias registradas (Tabela 1), confirmando sua boa sobrevivência em áreas de parques e reservas de perímetros urbanos, conforme observado por Brown Jr. \& Freitas (2002).

Durante realização de trabalhos anteriores de observação de borboletas no campus Camobi (Lemes et al., dados não publicados) foi registrada a presença de pelo menos mais 12 espécies de borboletas (Tabela 1), aumentando a riqueza do campus para 101 espécies. A maioria delas foi observada no Jardim Botânico, área que parece apresentar bons recursos de habitat para borboletas, com exceção do papilionídeo Parides anchises nephalion (Godart, 1819) e dos ninfalídeos Mechanitis lysimnia lysimnia (Fabricius, 1793) e Methona themisto (Hübner, 1818).

A maioria das espécies acidentais registradas no campus UFSM pertence às famílias Nymphalidae e Hesperiidae (Tabela 1), dentre elas Actinote mamita mamita (Burmeister,1861) (Nymphalidae), Lycas argentea (Hewitson, 1866) e Nyctelius nyctelius nyctelius (Latreille, [1824]) (Hesperiidae) que também foram consideradas escassas ou pouco comuns em outras localidades (Brown Jr. 1992). Por outro lado, espécies consideradas acidentais no presente trabalho, como os licenídeos Rekoa palegon Cramer, 1780 e Panthiades hebraeus (Hewitson, 1867) e os ninfalídeos Actinote pellenea Hübner, [1821] e Hypanartia lethe (Fabricius, 1793), são referidas como comuns e até mesmo muito comuns em outros ambientes (Brown Jr. 1992). Grande parte das espécies constantes da área do campus, incluindo as cinco espécies de borboletas mais abundantes (já mencionadas anteriormente) também está frequentemente associada a diversos tipos de ambiente (Brown Jr. 1992). Nenhuma espécie das famílias Lycaenidae e Riodinidae foi considerada constante no presente estudo e isso pode estar relacionado ao período relativamente curto de amostragem e também à conhecida dificuldade de amostragem dessas famílias (Brown Jr. 1992, Brown Jr. \& Freitas 1999). Seis espécies, no entanto, foram consideradas acessórias na área do presente trabalho, como os licenídeos Parrhasius orgia (Hewitson, 1867), espécie migratória (Brown Jr. 1992), Strymon eurytulus (Hübner, [1819]), pouco comum em campos abertos (Brown Jr. 1992), e o riodinídeo Riodina lycisca (Hewitson, [1853]), considerado comum em localidades do estado de São Paulo (Brown Jr. 1992).

Apesar do conceito de "singleton" estar às vezes associado ao de espécie rara, alguns daqueles amostrados no campus, como os hesperídeos Heliopetes alana (Reakirt, 1868), Nyctelius nyctelius nyctelius (Latreille, [1824]), o pierídeo Eurema albula (Cramer, 1775) e o licenídeo Panthiades hebraeus (Hewitson, 1867), (Tabela 1), são considerados comuns em áreas abertas ou florestas perturbadas (Brown Jr. 1992). Por outro lado, espécies como o papilionídeo Heraclides hectorides (Esper, 1794), encontrado no interior ou borda de mata na região de Santa Maria (Schwartz \& Di Mare 2001) provavelmente constituam-se em espécies raras num ambiente fortemente antropizado como o campus Camobi. Assim, espécies consideradas raras em um determinado local podem não ser em outra área, devido a diferentes disponibilidades de recursos alimentares, plantas hospedeiras, ou fatores microclimáticos, dentre outros fatores ambientais (Brown Jr. \& Freitas 2002).
Os valores de diversidade e dominância calculados para o campus, de modo geral, são semelhantes aos apresentados por Dessuy $\&$ Morais (2007), cujos maiores valores de diversidade podem estar relacionados à maior riqueza de espécies e abundância de indivíduos registrados nas áreas estudadas de Floresta Estacional Decidual.

Os menores valores de riqueza e abundância registrados em setembro de 2005 (Figura 1, Tabelas 2 e 3), época da primeira ocasião amostral, foram em parte decorrentes de um esforço amostral reduzido, (4 horas), causado por condições meteorológicas adversas. Esse mês registrou temperatura média $\left(14,8{ }^{\circ} \mathrm{C}\right)$ e precipitação acumulada mensal (212,5 mm) atípicas (Fonte: Estação Meteorológica do Departamento de Fitotecnia, CCR, UFSM), pois as normais climatológicas para Santa Maria (período 1961-1990) correspondem a $16,2{ }^{\circ} \mathrm{C}$ e $153,6 \mathrm{~mm}$, respectivamente (Figura 3). Por outro lado, nessa mesma ocasião amostral, foram registrados cerca de $90 \%$ (11) dos indivíduos de Actinote (Nymphalidae) identificados no presente estudo, os quais estavam reunidos em um arbusto florido de Asteraceae, comportamento característico dessas borboletas (Brown Jr. 1992). Ainda em relação às condições meteorológicas, o período de novembro de 2005 a agosto de 2006 registrou valores de precipitação bem abaixo das normais, com exceção de janeiro de 2006 (Figura 3). Segundo Brown Jr. (1992) a reprodução de borboletas, em geral associada a sazonalidade regional, ocorre normalmente de outubro a março, seguindo-se então uma diapausa das espécies até o próximo verão. No presente estudo, apesar da menor precipitação ter provavelmente influenciado os recursos ambientais, as maiores riquezas e abundâncias de borboletas foram registradas nas estações do verão e estenderam-se até o outono, com destaque para o mês de maio (quinta ocasião amostral) que apresentou a maior abundância de indivíduos registrada, principalmente nas famílias Hesperiidae e Nymphalidae (Tabela 3). Provavelmente este padrão de sazonalidade das borboletas registradas no campus da UFSM, encontrado também em parte da região sudeste e sul do Brasil (Brown Jr. 1972), está relacionado à existência de uma maior oferta de alimentos e plantas para oviposição (Brown Jr. \& Freitas 1999) do local.

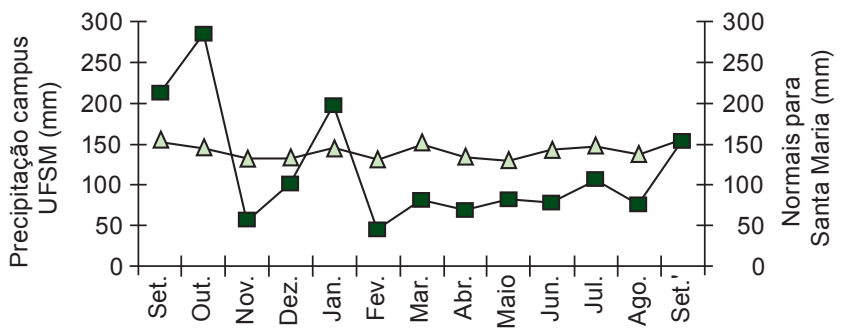

- Precipitação campus UFSM $\triangle-$ Normais para Santa Maria

Figura 3. Precipitação média mensal no campus Camobi da Universidade Federal de Santa Maria, Santa Maria, Rio Grande do Sul, Brasil, de setembro de 2005 (set) a setembro de 2006 (set') (Fonte: Estação Meteorológica do Departamento de Fitotecnia, CCR, UFSM) e normais de precipitação de Santa Maria, RS, com dados obtidos de 1961 a 1990 (Fonte: Instituto Nacional de Meteorologia- INMET, Oitavo Distrito de Meteorologia - $8^{\circ}$ DISME; Estação Climatológica Principal de Santa Maria, RS; Latitude $29^{\circ} 42^{\prime}$ e Longitude $53^{\circ} 42^{\prime}$ ).

Figure 3. Monthly mean precipitation on Universidade Federal de Santa Maria campus area of Camobi, Santa Maria, Rio Grande do Sul, Brazil, from September 2005 (Set) to September 2006 (Set') (Source: Estação Meteorológica do Departamento de Fitotecnia, CCR, UFSM) and historical precipitation on Santa Maria, RS, from 1961 to 1990 (Source: Instituto Nacional de MeteorologiaINMET, Oitavo Distrito de Meteorologia - $8^{\circ}$ DISME; Estação Climatológica Principal de Santa Maria, RS; Latitude 29 $9^{\circ}$ ' and Longitude 5342'). 
Apesar do pequeno número de horas amostradas e do relativo grau de impactação, o campus Camobi da Universidade Federal de Santa Maria possui fauna de borboletas expressiva, demonstrando que ainda pode oferecer recursos para a sobrevivência das espécies ali encontradas. Inventários de répteis e mamíferos, realizados no mesmo local, mostraram a presença de espécies típicas de campo, áreas abertas, e algumas consideradas raras ou ameaçadas (Santos et al. 2005, 2008), reforçando a importância dessa área de estudo.

Recomendamos a continuação de estudos visando caracterizar essa área como refúgio urbano de fauna associada ao bioma Pampa, cuja biodiversidade é pouco conhecida e bastante ameaçada. Para manutenção desta fauna e ambientes associados, faz-se necessária a efetivação de áreas de preservação legal que incorporem este bioma.

\section{Agradecimentos}

Às colegas do Laboratório de Interações Inseto-Planta, Camila D. Ritter e Renata Lemes, pelo auxílio no campo e aos pesquisadores do Laboratório de Bioecologia de Insetos da UFRGS pela ajuda nas primeiras identificações. Aos Doutores Olaf H. H. Mielke (UFPR), Ronaldo B. Francini (Unisantos), Robert K. Robbins (Smithsonian Institution), Curtis Callaghan e Rocco A. DiMare (UFSM) pelas preciosas identificações de Hesperiidae, Actinote, Lycaenidae, Riodinidae e Satyrinae. Às Mestres Ana Luíza G. P. Marks, Mônica B. Dessuy e dois revisores anônimos pelas valiosas sugestões e correções deste trabalho.

\section{Referências Bibliográficas}

BECCALONI, G.W. \& GASTON, K.J. 1995. Predicting species richness of neotropical forest butterflies: Ithomiinae (Lepidoptera, Nymphalidae) as indicator. Biol. Conserv. 71:77-86.

BIEZANKO, C.M. 1949. Acraeidae, Heliconidae e Nymphalidae de Pelotas e arredores. Pelotas, Livraria do Globo, 16p.

BIEZANKO, C.M. 1960a. III. Danaidae et Ithomidae da zona sueste do Rio Grande do Sul. Arquivos de entomologia, série A:1-6.

BIEZANKO, C.M. 1960b. III. Danaidae et Ithomidae da zona missioneira do Rio Grande do Sul. Arquivos de entomologia, série B:1-6.

BIEZANKO, C. M. 1960c. IV. Satyridae, Morphidae et Brassolidae da zona sueste do Rio Grande do Sul. Arquivos de entomologia, série A:1-13.

BIEZANKO, C.M. 1960d. IV. Satyridae, Morphidae et Brassolidae da zona missioneira do Rio Grande do Sul. Arquivos de entomologia, série B:1-10.

BIEZANKO, C.M. 1963. VI. Hesperiidae da zona sueste do Rio Grande do Sul. Arquivos de entomologia, série A: -25.

BIEZANKO, C.M. \& MIELKE, O.H. 1973. Contribuição ao estudo faunístico dos Hesperiidae americanos. IV. Espécies do Rio Grande do Sul, Brasil, com notas taxonômicas e descrições de espécies novas (Lepidoptera). Acta Biológica Paranaense 2(1-4):51-102.

BIEZANKO, C. M., MIELKE, O. H. \& WEDDERHOFF. 1978. Contribuição ao estudo faunístico dos Riodinidae do Rio Grande do Sul, Brasil (Lepidoptera). Acta Biológica Paranaense 7(1-4):7-22.

BROWN-Jr., K.S. 1972. Maximizing daily butterfly counts. J. Lepid. Soc. 26(3):183-196.

BROWN-Jr., K.S. 1992. Borboletas da Serra do Japi: diversidade, habitats, recursos alimentares e variação temporal. In História natural da Serra do Japi: ecologia e preservação de uma área florestal no sudeste do Brasil (L. P. C. Morellato, org.). UNICAMP, Campinas, p.142-186.

BROWN-Jr., K.S. \& FREITAS, A.V.L. 1999. Lepidoptera. In Biodiversidade do estado de São Paulo, Brasil: síntese do conhecimento ao final do século XX, 5: invertebrados terrestres (C. R. F. Brandão \& E. M. Cancello, org.). FAPESP, São Paulo, p. 227-243.

BROWN-Jr., K. S. \& FREITAS, A.V.L. 2002. Butterfly communities of urban forest fragments in Campinas, São Paulo, Brazil: structure, in- stability, environmental correlates, and conservation. J. Insect Conserv. 6(4):217-231.

CANALS, G.R. 2000. Mariposas bonaerenses. Ed. L.O.L.A., Buenos Aires.

CANALS, G.R. 2003. Mariposas de Misiones. Ed. L.O.L.A., Buenos Aires.

COLLIER, N.; MACKAI, D.A.; BENKENDORFF, K.; AUSTIN, A.D. \& CARTHEW, S.M. 2006. Butterfly communities in south australian urban reserves: estimating abundance and diversity using the Pollard walk Austral Ecol. 31:282-290.

DAMBROS, V.S.; EISINGER, S. M. \& CANTO-DOROW, T.S. 2004. Leguminosae do Campus da Universidade Federal de Santa Maria, Rio Grande do Sul, Brasil. Ciência e Natura 26(2):43-60.

DESSUY, M.B. \& MORAIS, A.B.B. 2007. Diversidade de borboletas (Lepidoptera: Papilionoidea e Hesperioidea) em fragmentos de floresta estacional decidual em Santa Maria, Rio Grande do Sul, Brasil. Rev. Bras. Zool. 24(1):108-120.

D' ABRERA, B. 1988. Butterflies of the neotropical region. Part V. Nymphalidae (conc.) \& Satiridae. Hill House, Victoria.

D' ABRERA, B. 1994. Butterflies of the neotropical region. Part VI. Riodinidae. Hill House, Victoria.

D' ABRERA, B. 1995. Butterflies of the neotropical region. Part VII. Lycaenidae. Hill House, Victoria.

De VRIES, P.J. 1987. The butterflies of Costa Rica and their natural history. Papilionidae, Pieridae, Nymphalidae. Princeton University Press, Princeton.

De VRIES, P.J. 1997. The butterflies of Costa Rica and their natural history. Vol. II: Riodinidae. Princeton University Press, Princeton.

ETHUR, L.Z.; EISINGER, S. M. \& RITTER, M. R. 1995. Levantamento de plantas invasoras no campus da UFSM- Santa Maria, RS- Magnoliopsida. Ciência e Natura 17:65-73.

FAHRIG, L. 2003. Effects of habitat fragmentation on biodiversity. Ann. Rev. Ecol. Evol. Syst. 34: 487-515.

FREITAS, A.V.L.; LEAL, I.R.; PRADO, M. H. \& IANNUZZI, L. 2006. Biologia da conservação: essências. In Insetos como indicadores de conservação de paisagem (C. F. D. Rocha; H. G. Bergallo; M. Van Sluys \& M.A.S. Alves, eds.). Rima, São Carlos, p. 357- 384.

HEPPNER, J.B. 1991. Faunal regions and the diversity of Lepidoptera. Trop. Lep. 2(1):1-85.

ISERHARD, C. A. \& ROMANOWSKI, H. P. 2004. Lista de espécies de borboletas (Lepidoptera, Papilionoidea e Hesperioidea) da região do vale do rio Maquiné, Rio Grande do Sul, Brasil. Rev. Bras. Zool. 21(3):649-662.

KAMINSKI, M.L.J. 1992. Avaliação quali-quantitativa de coleções de insetos de Santa Maria e arredores. Monografia de especialização em Biologia. Universidade Federal de Santa Maria, Santa Maria, Rio Grande do Sul.

KOH, L.P. \& SODHI, N.S. 2004. Importance of reserves, fragments, and parks for butterfly conservation in a tropical urban landscape. Ecol. Appl. 14(6):1695-1708.

KRÜGER, C.P. \& SILVA, E.J.E. 2003. Papilionoidea (Lepidoptera) de Pelotas e seus arredores, Rio Grande do Sul, Brasil. Entomología y Vectores 10(1):31-45.

LAMAS, G. (ed.). 2004. Checklist: Part 4A. Hesperioidea-Papilionoidea. In Atlas of Neotropical Lepidoptera. Vol. 5A. (J.B. Heppner, ed.). Association for Tropical Lepidoptera/Scientific Publishers, Gainesville,

LINK, D., BIEZANKO, C.M., TARRAGÓ, M.F. \& CARVALHO, S. 1977. Lepidoptera de Santa Maria e arredores. I: Papilionidae e Pieridae. Rev. Centro Ciências Rurais 7(4):381-389.

LINK, D., BIEZANKO, C.M., TARRAGÓ, M.F. \& CARVALHO, S. 1980. Lepidoptera de Santa Maria e arredores. II: Morphidae e Brassolidae. Rev. Centro Ciências Rurais 10(2):191-195.

MARCHIORI, M.O. \& ROMANOWSKI, H. P. 2006a. Species composition and diel variation of a butterfly taxocene (Lepidoptera, Papilionoidea and Hesperioidea) in a restinga forest at Itapuã State Park, Rio Grande do Sul, Brazil. Rev. Bras. Zool. 23(2):443-454.

MARCHIORI, M.O. \& ROMANOWSKI, H.P. 2006b. Borboletas (Lepidoptera, Papilionoidea e Hesperioidea) do Parque Estadual do 
Espinilho e entorno, Rio Grande do Sul, Brasil. Rev. Bras. Zool. 23(4):1029-1037.

MIELKE, O.H.H. 1979. Contribuição ao estudo faunístico dos Hesperiidae americanos. V. Nota suplementar às espécies de Pyrrhopyginae e Pyrginae do Rio Grande do Sul, Brasil (Lepidoptera). Acta Biológica Paranaense 8/9:7-17. [1980].

MIELKE, O.H.H. 2005. Catalogue of the american Hesperioidea: Hesperiidae (Lepidoptera). Sociedade Brasileira de Zoologia, Curitiba, 6v.

MORAIS, A.B.B., ROMANOWSKI, H.P., ISERHARD, C.A., MARCHIORI, M.O.O. \& SEGUÍ, R. 2007. Mariposas del sur de Sudamérica (Lepidoptera: Hesperioidea y Papilionoidea). Revista Ciência \& Ambiente 35:29-46.

NOVOTNÝ, V. \& BASSET, Y. 2000. Rare species in communities of tropical insect herbivores: pondering the mistery of singletons. Oikos 89:564-572.

PAIM, A.C. \& DI MARE, R.A. 2002. Ecologia de Papilionidae. I: Parâmetros biológicos e demográficos de Parides agavus (Papilioninae, Troidini) no sul do Brasil. Biociências 10(2):33-48.

PAZ, A.L.G. 2005. Levantamento da diversidade de borboletas (Lepidoptera: Papilionoidea e Hesperioidea) na Serra do Sudeste do RS. Dissertação de mestrado, Universidade Federal do Rio Grande do Sul, Porto Alegre.

PEREIRA, P.R.B.,GARCIA NETTO, L.R., BORIM, C. J. A. \& SARTORI, M. G.B. 1989. Contribuição à geografia física do município de Santa Maria: unidades de paisagem. Geografia Ensino \& Pesquisa 2:37-68.

QUADROS, F.C., DORNELES, A.L. \& CORSEUIL, E. 2004. Ninfalídeos (Lepidoptera, Nymphalidae) ocorrentes no norte da planície costeira do Rio Grande do Sul, Brasil. Biociências 12(2):147-164.

SANTOS, T.G., KOPP, K.A., SPIES, M.R., TREVISAN, R. \& CECHIN, S. Z. 2005. Répteis do campus da Universidade Federal de Santa Maria, RS, Brasil. Biota Neotrop. 5(1): http://www.biotaneotropica.org.br/v5n1/pt/a bstract?inventory+BN02705012005 (último acesso em 15/03/2007)
SANTOS, T.G., SPIES, M.R., KOPP, K. A., TREVISAN, R. \& CECHIN, S.Z. 2008. Mamíferos do campus da Universidade Federal de Santa Maria, Rio Grande do Sul, Brasil. Biota Neotrop. 8(1): http://www. biotaneotropica.org.br/v8n1/pt/abstract?article+BN00508012008 (último acesso em 07/01/2008)

SCHWARTZ, G. \& DI MARE, R. A. 2001. Diversidade de quinze espécies de borboletas (Lepidoptera, Papilionidae) em sete comunidades de Santa Maria, RS. Ciência Rural 31(1):49-55.

TESTON, J.A. \& CORSEUIL, E. 2001. Ninfalídeos (Lepidoptera, Nymphalidae) ocorrentes no Rio Grande do Sul, Brasil. Parte I. Danainae e Ithomiinae. Biociências 9(1):51-61.

TESTON, J.A. \& CORSEUIL, E. 2002a. Borboletas (Lepidoptera, Rhopalocera) ocorrentes no Centro de Pesquisas e Conservação da Natureza Pró-Mata. 3. Nymphalidae. Divulgação do Museu de Ciência e Tecnologia 7:1-208

TESTON, J.A. \& CORSEUIL, E. 2002b. Ninfalídeos (Lepidoptera, Nymphalidae) ocorrentes no Rio Grande do Sul, Brasil. Parte II. Brassolinae e Morphinae. Biociências 10(1):75-84.

TYLER, H.A.; BROWN JR. K. S. \& WILSON, K. H. 1994. Swallowtail butterflies of the americas: a study in biological dynamics, ecological diversity, biosystematics, and conservation. Scientific Publishers, Gainesville.

WAHLBERG, N., BRABY, M. F., BROWER, A.V.Z., JONG, R., LEE, M., NYLIN, S., PIERCE, N.E., SPERLING, F. A. H., VILA, R., WARREN, A.D. \& ZAKHAROV, E. 2005. Synergistic effects of combining morphological and molecular data in resolving the phylogeny of butterflies and skippers. Proc. R. Soc. B. 272:1577-1586.

Recebido em 05/11/07 Versão reformulada recebida em 18/01/08 Publicado em 01/02/08 